\title{
ELASTIC BUCKLING OF A TRIANGULAR FRAME SUBJECT TO IN-PLANE TENSION
}

\author{
KRZYSZTOF MAGNUCKI \\ Poznan University of Technology, Institute of Applied Mechanics, Poznań, Poland \\ e-mail: krzysztof.magnucki@put.poznan.pl \\ SzyMON Milecki \\ Institute of Rail Vehicles, TABOR, Poznań, Poland
}

\begin{abstract}
The paper is devoted to elastic buckling of a symmetrical triangular frame under tensile in-plane load. Three mathematical models of the triangular frame are formulated. The first model deals with the pre-buckling state, the second one with the in-plane buckling state, and the third one with the lateral buckling state of the frame. The FEM-numerical model of the frame is formulated and the critical loads are calculated. The comparison of the results obtained in the analytical and numerical-FEM analysis is presented in tables and graphs in figures.
\end{abstract}

Keywords: triangular frame, elastic buckling, critical load, mathematical model

\section{Introduction}

The theoretical basis of buckling problems of structures is elaborated in many papers and monographs. Horne and Merchant (1965) described the problem of stability of frames. Thompson and Hunt (1973) presented a general theory of elastic stability of structures. Budiansky (1974) presented the theory of buckling and post-buckling behaviour of elastic structures. Chen and Liu (1987) described the theory of stability and implementation for structures. Bažant and Cedolin (1991) presented an extensive review of stability problems of structures: columns, frames, thin-walled beams, plates and shells. Simitses and Hodges (2006) presented the fundamentals of structural stability for columns, beams, rings and arches. Van der Heijden (2008) described the elastic stability of solids and structures formulated and studied by professor W.T. Koiter. Kaveh and Salimbahram (2007) presented a methodology for efficient calculation of buckling loads for symmetric rectangular frame structures. Şakar et al. (2012) presented FEM study of dynamic stability of a multi-span rectangular frame subjected to periodic loading.

The problem of triangular frame stability has been presented in literature only in several publications. For example, Magnucki and Milecki (2012) presented flat elastic buckling of the brake triangle in freight wagons, while Sobaś (2010) described the strength problems, especially the fatigue strength of the brake triangles.

The subject of theoretical study presented in this paper is a symmetrical triangular frame under tensile in-plane load (Fig. 1). The vertex $C$ of the frame is fixed, whereas the vertexes $A$ and $B$ are simply supported in the plane of the triangular frame.

The arms of length $L_{1}$ are connected with a cross-beam of length $L_{2}$. The cross-section of the arms is rectangular, while the cross-section of the cross-beam is a circular ring (Fig. 2). 


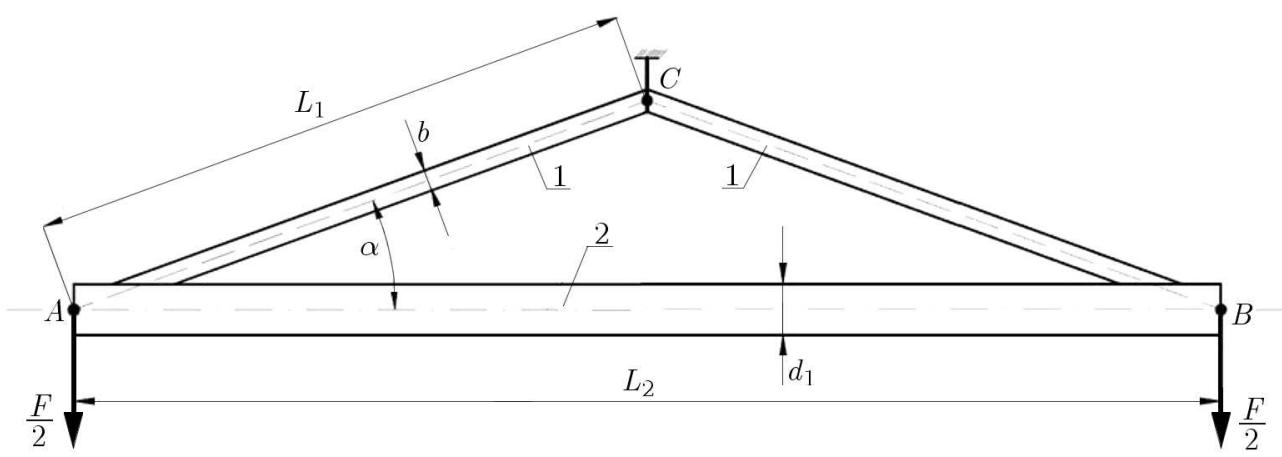

Fig. 1. Scheme of the symmetrical triangular frame with the load
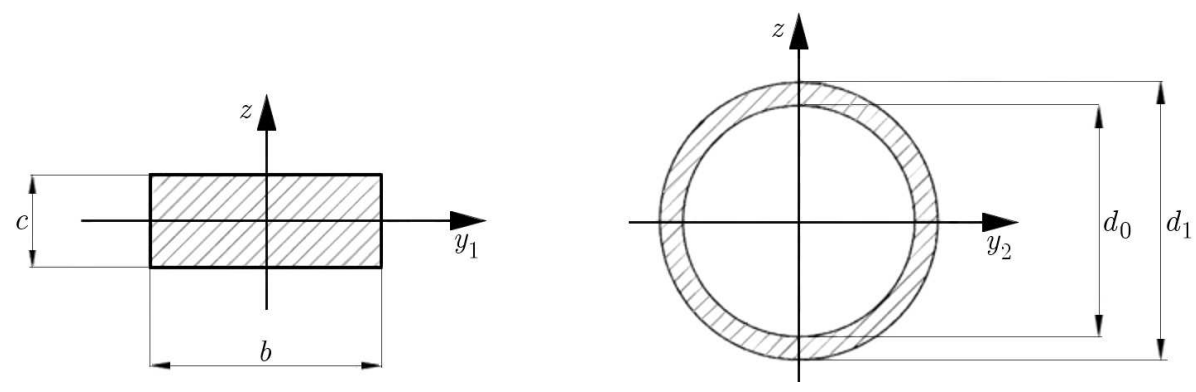

Fig. 2. Cross-section of the arms -1 and cross-beam -2

\section{Mathematical models}

\subsection{Pre-buckling state}

A half of the symmetrical triangular frame with the load $F$, internal normal force $N_{2}$ and bending moment $M_{2}$ for the pre-buckling state is shown in Fig. 3.

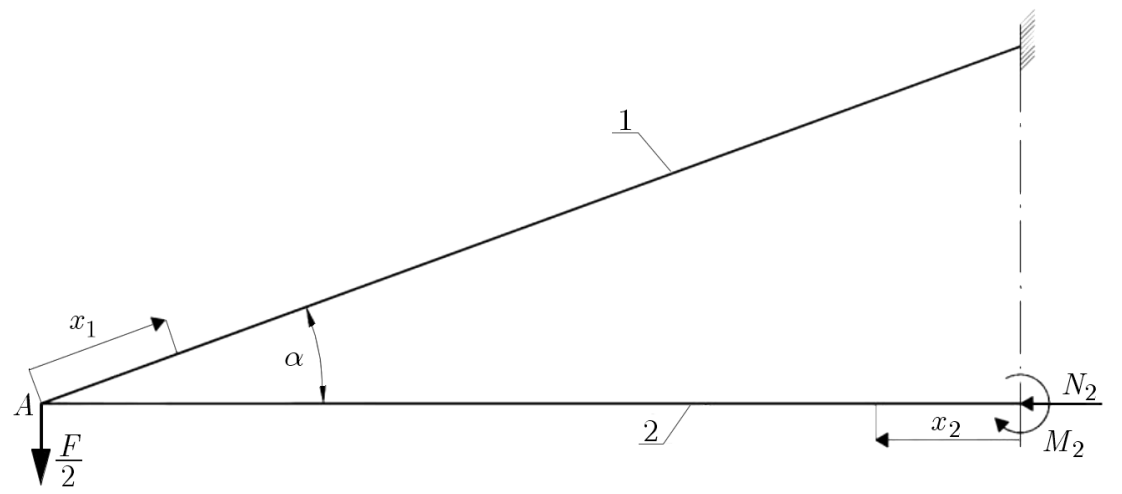

Fig. 3. Scheme of a half of the frame for the pre-buckling state

The normal forces $N\left(x_{i}\right)$ and bending moments $M_{b}\left(x_{i}\right)$ in the elements of the frame:

- $\operatorname{arm}-1$

$$
N\left(x_{1}\right)=N_{1}=N_{2} \cos \alpha+\frac{1}{2} F \sin \alpha \quad M_{b}\left(x_{1}\right)=\left(N_{2} \sin \alpha-\frac{1}{2} F \cos \alpha\right) x_{1}+M_{2}
$$

- cross-beam - 2

$$
N\left(x_{2}\right)=N_{2} \quad M_{b}\left(x_{2}\right)=M_{2}
$$


The elastic strain energy of the frame is

$$
\begin{aligned}
U_{\varepsilon} & =\frac{1}{2 E A_{1}} \int_{0}^{L_{1}}\left[N\left(x_{1}\right)\right]^{2} d x_{1}+\frac{1}{2 E J_{z}^{(1)}} \int_{0}^{L_{1}}\left[M_{b}\left(x_{1}\right)\right]^{2} d x_{1}+\frac{1}{2 E A_{2}} \int_{0}^{L_{2} / 2}\left[N\left(x_{2}\right)\right]^{2} d x_{2} \\
& +\frac{1}{2 E J_{z}^{(2)}} \int_{0}^{L_{2} / 2}\left[M_{b}\left(x_{2}\right)\right]^{2} d x_{2}
\end{aligned}
$$

where $A_{1}=b c, A_{2}=\pi\left(d_{1}^{2}-d_{0}^{2}\right) / 4$ are the areas of the cross-sections of the arm and the crossbeam, $J_{z}^{(1)}=b c^{3} / 12, J_{z}^{(2)}=\pi\left(d_{1}^{4}-d_{0}^{4}\right) / 64$ - inertia moments of the cross-sections of the arm and the cross-beam, $E$ - Young's modulus.

Taking into account the theorem of Menabrea, two conditions are formulated

$$
\begin{aligned}
& \frac{\partial U_{\varepsilon}}{\partial N_{2}}=\frac{1}{A_{1}} \int_{0}^{L_{1}} N\left(x_{1}\right) \cos \alpha d x_{1}+\frac{1}{J_{z}^{(1)}} \int_{0}^{L_{1}} M_{b}\left(x_{1}\right) x_{1} \sin \alpha d x_{1}+\frac{1}{A_{2}} \int_{0}^{L_{2} / 2} N_{2} d x_{2}=0 \\
& \frac{\partial U_{\varepsilon}}{\partial M_{2}}=\frac{1}{J_{z}^{(1)}} \int_{0}^{L_{1}} M_{b}\left(x_{1}\right) d x_{1}+\frac{1}{J_{z}^{(2)}} \int_{0}^{L_{2} / 2} M_{2} d x_{2}=0
\end{aligned}
$$

Thus, after integration, two equations are obtained

$$
\alpha_{11} \frac{M_{2}}{L_{2}}+\alpha_{12} N_{2}=\frac{1}{2} \beta_{1} F \quad \alpha_{21} \frac{M_{2}}{L_{2}}+\alpha_{22} N_{2}=\frac{1}{8} F
$$

from which

$$
N_{2}=\frac{1}{2} \frac{\alpha_{11}-4 \beta_{1} \alpha_{21} \alpha_{11}}{\alpha_{22}-\alpha_{12} \alpha_{21}} F \quad M_{2}=\frac{1}{8} \frac{4 \beta_{1} \alpha_{22}-\alpha_{12}}{\alpha_{11} \alpha_{22}-\alpha_{12} \alpha_{21}} F L_{2}
$$

where

$$
\begin{aligned}
& \alpha_{11}=\frac{A_{2} L_{2}^{2}}{4 J_{z}^{(1)}} \frac{\sin \alpha}{\cos ^{2} \alpha} \\
& \alpha_{12}=1+\frac{A_{2}}{A_{1}} \cos \alpha+\frac{A_{2} L_{2}^{2}}{12 J_{z}^{(1)}} \frac{\sin ^{2} \alpha}{\cos ^{3} \alpha} \\
& \beta_{1}=\left(\frac{A_{1} L_{2}^{2}}{12 J_{z}^{(1)} \cos ^{2} \alpha}-1\right) \frac{A_{2}}{A_{1}} \sin \alpha \\
& \alpha_{21}=1+\frac{J_{z}^{(1)}}{J_{z}^{(2)}} \cos \alpha \\
& \alpha_{22}=\frac{1}{4} \tan \alpha
\end{aligned}
$$

Thus, the normal tension force of arm (2.1) is as follows

$$
N_{1}=\frac{1}{2}\left(\frac{\alpha_{11}-4 \beta_{1} \alpha_{21}}{\alpha_{11} \alpha_{22}-\alpha_{12} \alpha_{21}} \cos \alpha+\sin \alpha\right) F
$$

The normal force $N_{2}(2.6)_{1}$ is a compressive force acting on the cross-beam which causes buckling of the frame.

\section{2. $\quad$ Flat buckling state - critical load}

The critical state for the flat buckling of the symmetrical triangular frame is demonstrated by symmetrical flexure of the cross-beam and the angles of rotation of the vertexes $A$ and $B$ in the $x y$ plane. A scheme of the flat buckling mode for one half of the frame is shown in Fig. 4. Short discussion of this buckling problem was presented by Bažant and Cedolin (1991).

The detailed scheme of the load and displacements for the arm and the cross-beam of the frame is presented in Fig. 5 . 


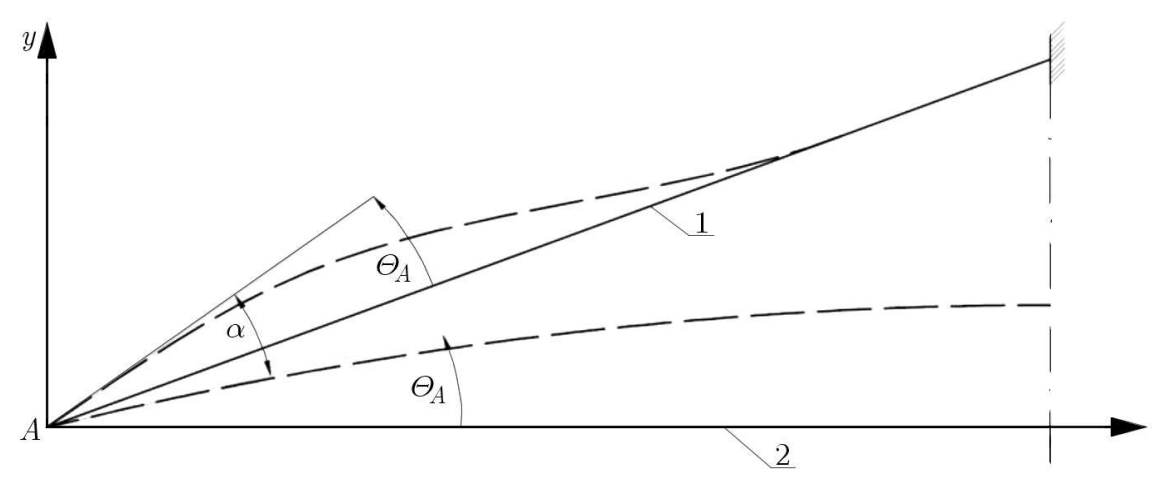

Fig. 4. Scheme of the flat buckling mode

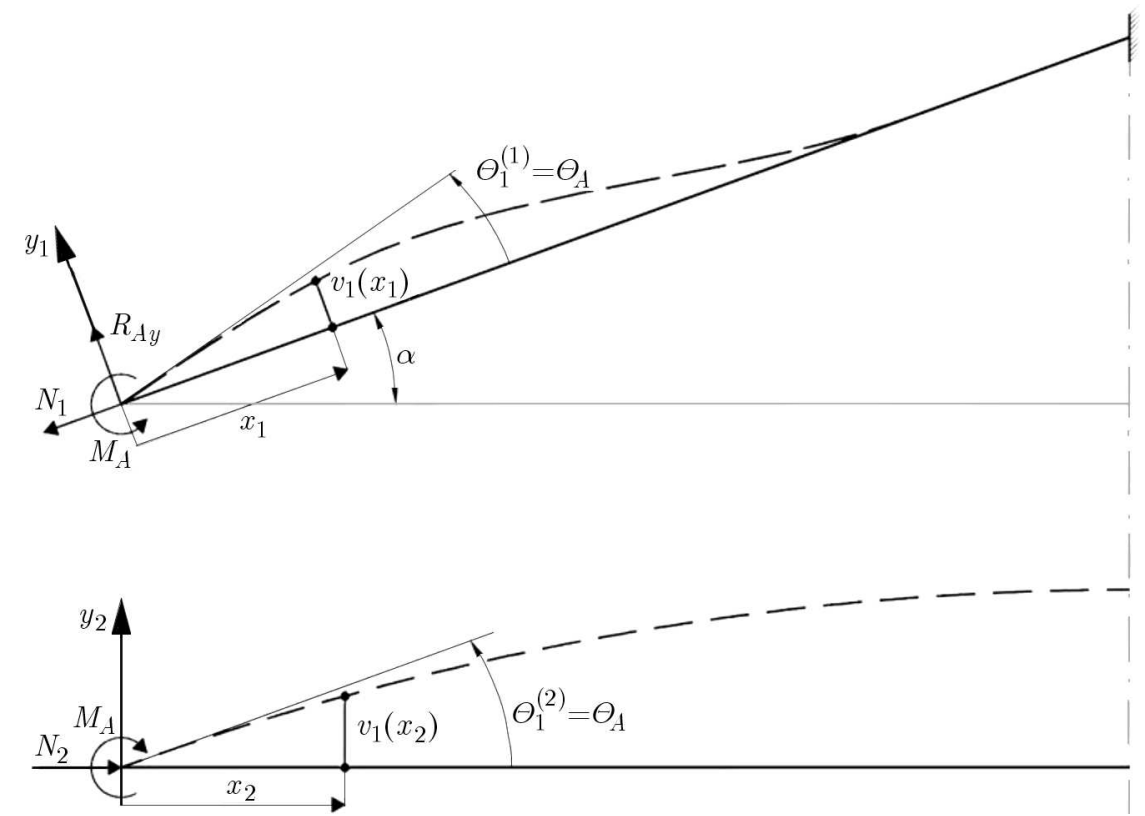

Fig. 5. Scheme of the load and displacements for the flat buckling state

The bending moment in the arm

$$
M_{b}\left(x_{1}\right)=M_{A}-N_{1} v_{1}\left(x_{1}\right)-R_{A y} x_{1}
$$

The differential equation of the bending line of the arm

$$
E J_{z}^{(1)} \frac{d^{2} v_{1}}{d x_{1}^{2}}-N_{1} v_{1}\left(x_{1}\right)=-M_{A}+R_{A y} x_{1}
$$

or

$$
\frac{d^{2} v_{1}}{d x_{1}^{2}}-k_{1 y}^{2} v_{1}\left(x_{1}\right)=-\frac{M_{A}}{E J_{z}^{(1)}}+\frac{R_{A y} x_{1}}{E J_{z}^{(1)}}
$$

where $k_{1 y}=\sqrt{N_{1} /\left(E J_{z}^{(1)}\right)}$ is a coefficient.

The solution of this equation is in the form

$$
v_{1}\left(x_{1}\right)=C_{1}^{(1)} \sinh \left(k_{1 y} x_{1}\right)+C_{2}^{(1)} \cosh \left(k_{1 y} x_{1}\right)+\frac{M_{A}}{N_{1}}-\frac{R_{A y}}{N_{1}} x_{1}
$$

where $C_{1}^{(1)}, C_{2}^{(1)}$ are the integration constants. 
The boundary conditions are as follows

$$
v_{1}(0)=0 \quad v_{1}\left(L_{1}\right)=\left.0 \quad \frac{d v_{1}}{d x_{1}}\right|_{L_{1}}=0
$$

from which

$$
C_{1}^{(1)}=\frac{M_{A}}{N_{1}} \frac{1+k_{1 y} L_{1} \sinh \left(k_{1 y} L_{1}\right)-\cosh \left(k_{1 y} L_{1}\right)}{k_{1 y} L_{1} \cosh \left(k_{1 y} L_{1}\right)-\sinh \left(k_{1 y} L_{1}\right)} \quad C_{2}^{(1)}=-\frac{M_{A}}{N_{1}}
$$

and the reaction

$$
R_{A y}=k_{1 y} M_{A}\left[\frac{1+k_{1 y} L_{1} \sinh \left(k_{1 y} L_{1}\right)-\cosh \left(k_{1 y} L_{1}\right)}{k_{1 y} L_{1} \cosh \left(k_{1 y} L_{1}\right)-\sinh \left(k_{1 y} L_{1}\right)} \cosh \left(k_{1 y} L_{1}\right)-\sinh \left(k_{1 y} L_{1}\right)\right]
$$

Thus, the angle of the $A$ vertex rotation in the $x y$ plane is

$$
\theta_{A}^{(1)}=\left.\frac{d v_{1}}{d x_{1}}\right|_{0}=\frac{M_{A} L_{1}}{E J_{z}^{(1)}} f_{1 y}\left(k_{1 y} L_{1}\right)
$$

where

$$
f_{1 y}\left(k_{1 y} L_{1}\right)=\frac{1}{k_{1 y} L_{1}}\left\{\sinh \left(k_{1 y} L_{1}\right)-\frac{1+k_{1 y} L_{1} \sinh \left(k_{1 y} L_{1}\right)-\cosh \left(k_{1 y} L_{1}\right)}{k_{1 y} L_{1} \cosh \left(k_{1 y} L_{1}\right)-\sinh \left(k_{1 y} L_{1}\right)}\left[\cosh \left(k_{1 y} L_{1}\right)-1\right]\right\}
$$

Similarly, the bending moment in the cross-beam is

$$
M_{b}\left(x_{2}\right)=-M_{A}+N_{2} v_{2}\left(x_{2}\right)
$$

The differential equation of the bending line

$$
\frac{d^{2} v_{2}}{d x_{2}^{2}}+k_{2}^{2} v_{2}\left(x_{2}\right)=\frac{M_{A}}{E J_{z}^{(2)}}
$$

where $k_{2}=\sqrt{N_{2} /\left(E J_{z}^{(2)}\right)}$ is a coefficient.

The solution of this equation is

$$
v_{2}\left(x_{2}\right)=C_{1}^{(2)} \sinh \left(k_{2} x_{2}\right)+C_{2}^{(2)} \cosh \left(k_{2} x_{2}\right)+\frac{M_{A}}{N_{2}}
$$

The boundary conditions

$$
v_{2}(0)=0 \quad v_{2}\left(L_{2}\right)=0
$$

from which the integration constants are

$$
C_{1}^{(2)}=-\frac{M_{A}}{N_{2}} \frac{1-\cos \left(k_{2} L_{2}\right)}{\sin \left(k_{2} L_{2}\right)} \quad C_{2}^{(2)}=-\frac{M_{A}}{N_{2}}
$$

Thus, the angle of the $A$ vertex rotation in the $x y$ plane is

$$
\theta_{A}^{(2)}=\left.\frac{d v_{2}}{d x_{2}}\right|_{0}=-\frac{M_{A} L_{2}}{E J_{z}^{(2)}} \frac{1-\cos \left(k_{2} L_{2}\right)}{k_{2} L_{2} \sin \left(k_{2} L_{2}\right)}
$$


The consistency condition for the angles of in-plane rotation of the vertex $A$ for the arm and cross-beam

$$
\theta_{A}^{(1)}=\theta_{A}^{(2)}
$$

from which the nonlinear algebraic equation is in the following form

$$
k_{J z z} f_{1 y}\left(k_{1 y} L_{1}\right)+2 \frac{1-\cos \left(k_{2} L_{2}\right)}{k_{2} L_{2} \sin \left(k_{2} L_{2}\right)}=0
$$

where $k_{J z z}=J_{z}^{(2)} /\left(J_{z}^{(1)} \cos \alpha\right)$ is a dimensionless parameter.

Taking into account the normal forces $N_{2}(2.6)_{1}$ and $N_{1}(2.7)$, the critical load from this equation is determined.

\subsection{Lateral buckling state - critical load}

The critical state for the lateral buckling of the triangular frame is demonstrated by symmetrical flexure of the cross-beam in the xz plane and the corresponding angles of rotation of the vertexes $A$ and $B$. A scheme of the lateral buckling mode for the half of the frame is shown in (Fig. 6).

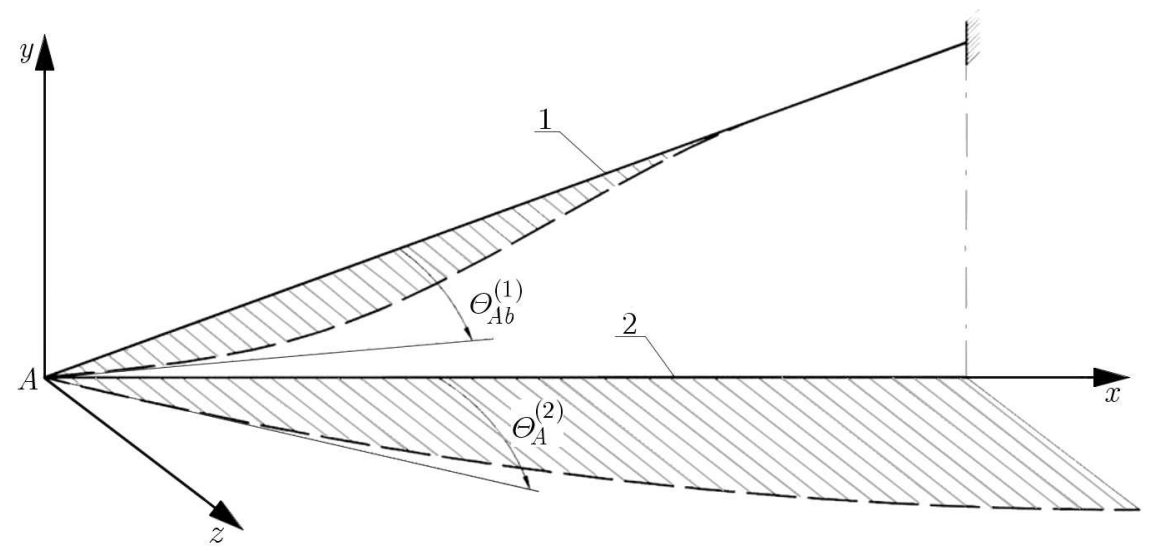

Fig. 6. Scheme of the lateral buckling mode

The flexure of the cross-beam in the xz plane causes bending and torsion of the arms. A scheme of the moments in the vertex $A$ is shown in Fig. 7 .

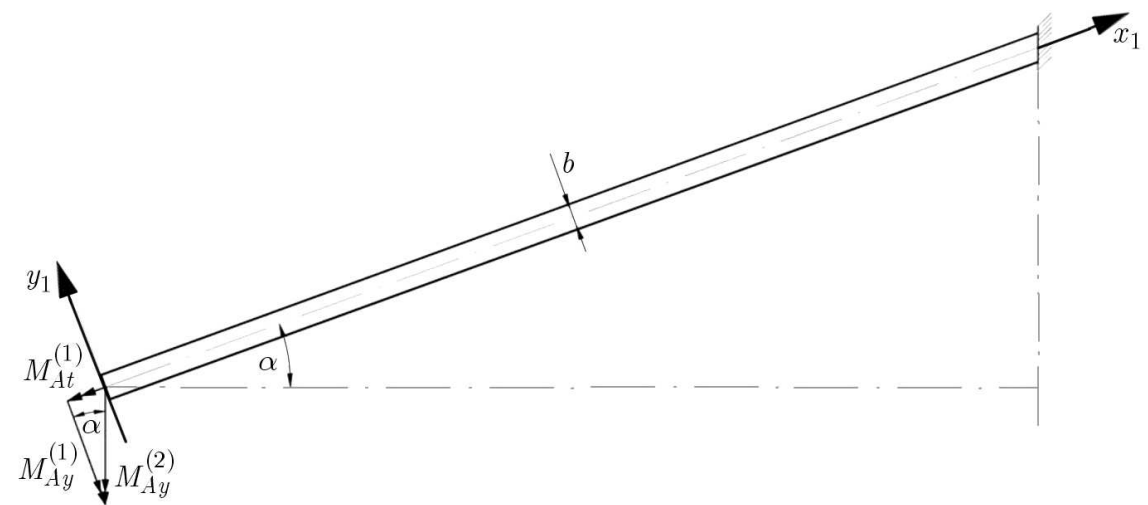

Fig. 7. Scheme of the moments in the vertex $A$ 
The bending moment $M_{A y}^{(1)}$ and torsional moment $M_{A t}^{(1)}$ in the vertex $A$ for the arm are

$$
M_{A y}^{(1)}=M_{A y}^{(2)} \cos \alpha \quad M_{A t}^{(1)}=M_{A y}^{(2)} \sin \alpha
$$

where $M_{A y}^{(2)}$ is the bending moment in the vertex $A$ for the cross-beam.

Analogically, the bending angle $\theta_{A b}^{(1)}$ and torsion angle $\theta_{A t}^{(1)}$ in the vertex $A$ for the arm are

$$
\theta_{A b}^{(1)}=\theta_{A}^{(2)} \cos \alpha \quad \theta_{A t}^{(1)}=\theta_{A}^{(2)} \sin \alpha
$$

where $\theta_{A}^{(2)}$ is the bending angle in the vertex $A$ for the cross-beam.

The detailed scheme of the load and displacements for the arm and the cross-beam of the frame is presented in Fig. 8 .

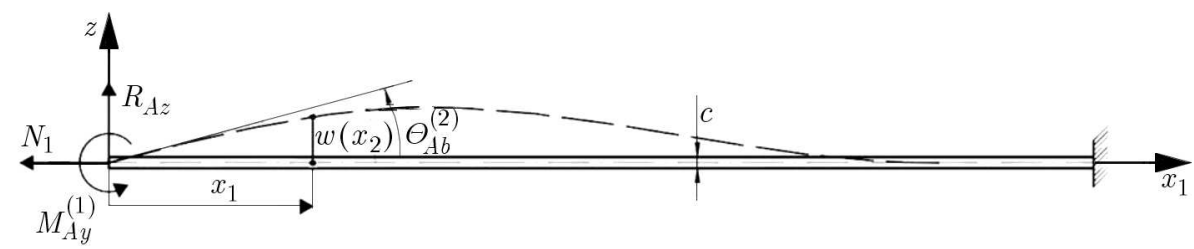

Fig. 8. Scheme of the load and displacements for lateral buckling state

The bending moment in the arm is

$$
M_{b}\left(x_{1}\right)=M_{A y}^{(1)}-N_{1} w_{1}\left(x_{1}\right)-R_{A z} x_{1}
$$

This bending problem is analogous to the bending of the arm for flat buckling state (10), thus the bending angle of the vertex $A$ is

$$
\theta_{A b}^{(1)}=\left.\frac{d w_{1}}{d x_{1}}\right|_{0}=\frac{M_{A y}^{(1)} L_{1}}{E J_{y}^{(1)}} f_{1 z}\left(k_{1 z} L_{1}\right)
$$

where

$$
f_{1 z}\left(k_{1 z} L_{1}\right)=\frac{1}{k_{1 z} L_{1}}\left\{\sinh \left(k_{1 z} L_{1}\right)-\frac{1+k_{1 z} L_{1} \sinh \left(k_{1 z} L_{1}\right)-\cosh \left(k_{1 z} L_{1}\right)}{k_{1 z} L_{1} \cosh \left(k_{1 z} L_{1}\right)-\sinh \left(k_{1 z} L_{1}\right)}\left[\cosh \left(k_{1 z} L_{1}\right)-1\right]\right\}
$$

and $k_{1 z}=\sqrt{N_{1} /\left(E J_{y}^{(1)}\right)}$ is a coefficient, $J_{y}^{(1)}=b^{3} c / 12$ - inertia moment of the cross-sections of the arm.

The torsion angle $\theta_{A t}^{(1)}$ in the vertex $A$

$$
\theta_{A t}^{(1)}=\frac{M_{A t}^{(1)} L_{1}}{G J_{t}^{(1)}}=2(1+\nu) \frac{M_{A t}^{(1)} L_{1}}{E J_{t}^{(1)}}
$$

where: $G=E /[2(1+\nu)]$ is the shear modulus of elasticity, $\nu$ - Poisson's ratio, and the torsional constant $J_{t}^{(1)}$ of the rectangular cross-section

- for $b \leqslant c$

$$
J_{t}^{(1)}=\mu b^{3} c \quad \mu=\frac{1}{3}-\left[0.178+0.153 \frac{b}{c}-0.138\left(\frac{b}{c}\right)^{2}\right]
$$

- for $c \leqslant b$

$$
J_{t}^{(1)}=\mu b c^{3} \quad \mu=\frac{1}{3}-\left[0.178+0.153 \frac{c}{b}-0.138\left(\frac{c}{b}\right)^{2}\right]
$$


The bending moment in the cross-beam is

$$
M_{b}\left(x_{2}\right)=-M_{A y}^{2}+N_{2} w_{2}\left(x_{2}\right)
$$

This bending problem is analogous to the bending of the cross-beam for flat buckling state (2.17), thus the bending angle of the vertex $A$ is

$$
\theta_{A}^{(2)}=\left.\frac{d w_{2}}{d x_{2}}\right|_{0}=-\frac{M_{A y}^{(2)} L_{2}}{E J_{z}^{(2)}} \frac{1-\cos \left(k_{2} L_{2}\right)}{k_{2} L_{2} \sin \left(k_{2} L_{2}\right)}
$$

Taking into account expressions (2.26) for the bending angle $\theta_{A b}^{(1)}$ and torsion angle $\theta_{A t}^{(1)}$, the consistency condition for the angles of the vertex $A$ is as follows

$$
\theta_{A b}^{(1)} \cos \alpha+\theta_{A t}^{(1)} \sin \alpha=\theta_{A}^{(2)}
$$

Substituting expressions (2.28) and (2.30) into equation (2.35) and making simple transformation, the nonlinear algebraic equation is obtained in the following form

$$
k_{J z y} f_{1 z}\left(k_{1 z} L_{1}\right) \cos ^{2} \alpha+2(1+\nu) k_{J z t} \sin ^{2} \alpha+2 \frac{1-\cos \left(k_{2} L_{2}\right)}{k_{2} L_{2} \sin \left(k_{2} L_{2}\right)}=0
$$

where: $k_{J z y}=J_{z}^{(2)} /\left(J_{y}^{(1)} \cos \alpha\right), k_{J z t}=J_{z}^{(2)} /\left(J_{t}^{(1)} \cos \alpha\right)$ are dimensionless parameters.

Taking into account the normal forces $N_{2}(2.6)_{1}$ and $N_{1}$ (2.7), the critical load from this equation is determined.

\subsection{Example of the triangular frame - values of the critical load}

An examplary calculation of the critical load is carried out for a symmetrical triangular frame: length of the cross-beam $L_{2}=1352 \mathrm{~mm}$, angle between the arms and the cross-beam $\alpha=\pi / 9$, diameters of the cross-section of the cross-beam $d_{0}=50 \mathrm{~mm}, d_{1}=60 \mathrm{~mm}$, area of the cross section of the arms $A_{1}=1000 \mathrm{~mm}^{2}$ and material constants $E=2 \cdot 10^{5} \mathrm{MPa}, \nu=0.3$. Models with different $b$ parameter have been investigated. Values of other parameters are the same as the parameters of the construction which is used in railway industry. The values of critical loads $F_{C R \text {, Flat }}^{(\text {Anal }}$ and $F_{C R \text {, Lat }}^{(\text {Anal })}$ have been calculated on the basis of non-linear algebraic equations (2.24) and (2.36), respectively. The results of calculations are specified in Table 1.

Table 1. Values of critical loads - analytical solution

\begin{tabular}{|c||c|c|c|c|c|c|c|c|}
\hline$b[\mathrm{~mm}]$ & 15 & 20 & 25 & 31.623 & 35 & 40 & 50 & 60 \\
\hline$F_{C R, \text { Flat }}^{(\text {Anal }}[\mathrm{kN}]$ & 360.6 & 403.0 & 448.2 & 510.0 & 541.5 & 587.1 & 672.5 & 747.8 \\
\hline$F_{\text {CR, Lat }}^{\text {Anal }}[\mathrm{kN}]$ & 363.9 & 394.6 & 403.4 & 399.2 & 388.7 & 377.1 & 357.1 & 342.8 \\
\hline
\end{tabular}

Flat buckling occurs when the width $b$ of the arm is small $(b \leqslant 18 \mathrm{~mm})$, and the lateral buckling occurs for greater width $(18 \mathrm{~mm} \leqslant b)$.

\section{Numerical FEM model}

\subsection{FEM model of the triangular frame}

The symmetrical triangular frame is a beam structure (Fig. 9), hence it is modeled with beam elements - system SolidWorks Simulation 2013 (Fig. 10). The model of the frame is defined in the rectangular coordinate system. The loading force is applied to the vertexes of the frame (Fig. 11). 


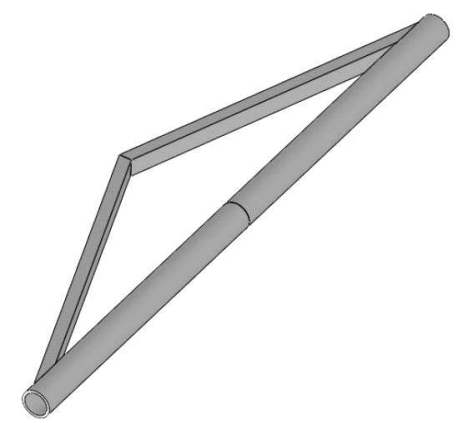

Fig. 9. Numerical model of triangular frame

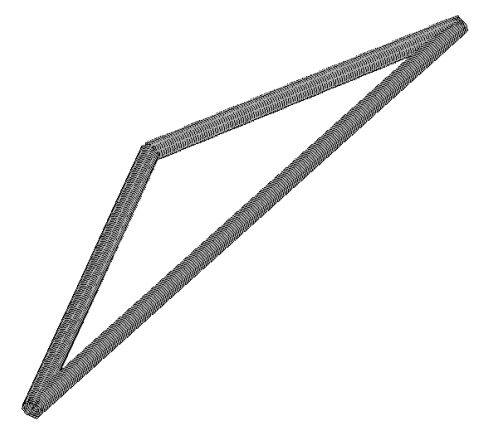

Fig. 10. Discretization of numerical model with the beam elements

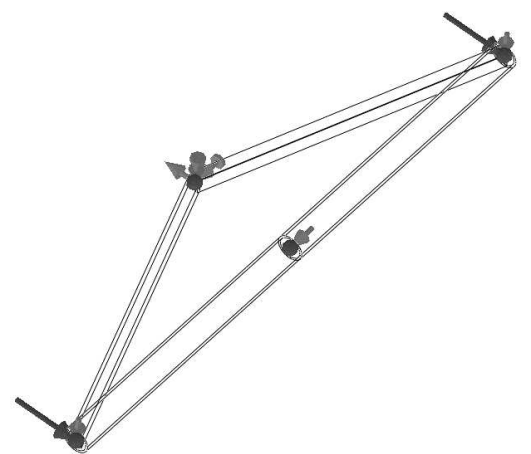

Fig. 11. Boundary conditions and loads of the numerical model

\subsection{Example of the triangular frame - values of the critical load}

An examplary calculation of the critical load is carried out for the symmetrical triangular frame: length of the cross-beam $L_{2}=1352 \mathrm{~mm}$, angle between arms and the cross-beam $\alpha=\pi / 9$, diameters of the cross-section of the cross-beam $d_{0}=50 \mathrm{~mm}, d_{1}=60 \mathrm{~mm}$, the area of the cross section of the arms $A_{1}=1000 \mathrm{~mm}^{2}$ and material constants $E=2 \cdot 10^{5} \mathrm{MPa}, \nu=0.3$. The values of critical loads $F_{C R, F l a t}^{(F E M)}$ and $F_{C R, \text { Lat }}^{(F E M)}$ are calculated on the basis of the finite element method. The results of calculations are specified in Table 2. An examplary FEM calculation of the triangular frame with dimension $b=25 \mathrm{~mm}$ is shown in Fig. 12.

Flat buckling, similarly to the analytical model, occurs when the width $b$ of the arm is small $(b \leqslant 18 \mathrm{~mm})$, and the lateral buckling occurs for greater width $(18 \mathrm{~mm} \leqslant b)$. 
Table 2. The values of critical loads - FEM solution

\begin{tabular}{|c||c|c|c|c|c|c|c|c|}
\hline$b[\mathrm{~mm}]$ & 15 & 20 & 25 & 31.623 & 35 & 40 & 50 & 60 \\
\hline$F_{C R, \text { Flat }}^{(F E M)}[\mathrm{kN}]$ & 353.7 & 390.7 & 435.6 & 492.4 & 520.9 & 562.1 & 638.2 & 704.7 \\
\hline$F_{\text {CR, Lat }}^{(F E M)}[\mathrm{kN}]$ & 361.6 & 393.5 & 400.5 & 393.2 & 385.5 & 373.4 & 352.7 & 338.6 \\
\hline
\end{tabular}

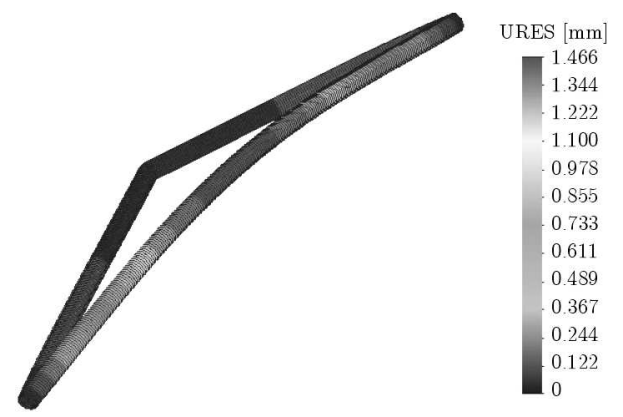

Fig. 12. Scheme of the FEM model of the triangular frame

\section{Comparison analysis}

The values of critical loads calculated analytically and numerically (FEM) are similar. The difference between them is less than 3\%. Comparison of these values is graphically shown for flat buckling (Fig. 13) and for lateral buckling (Fig. 14).

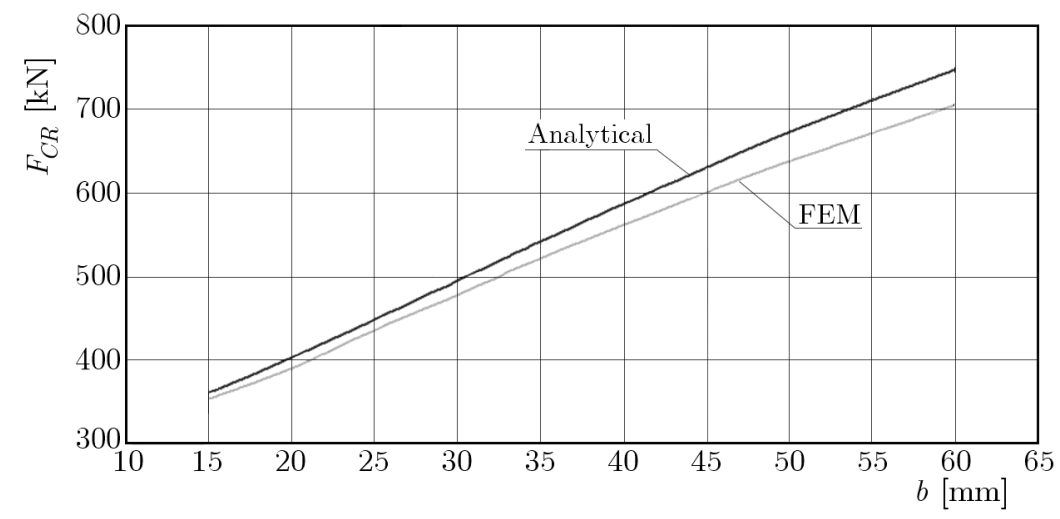

Fig. 13. Comparison of the critical values obtained from the analytical and numerical (FEM) methods for flat buckling

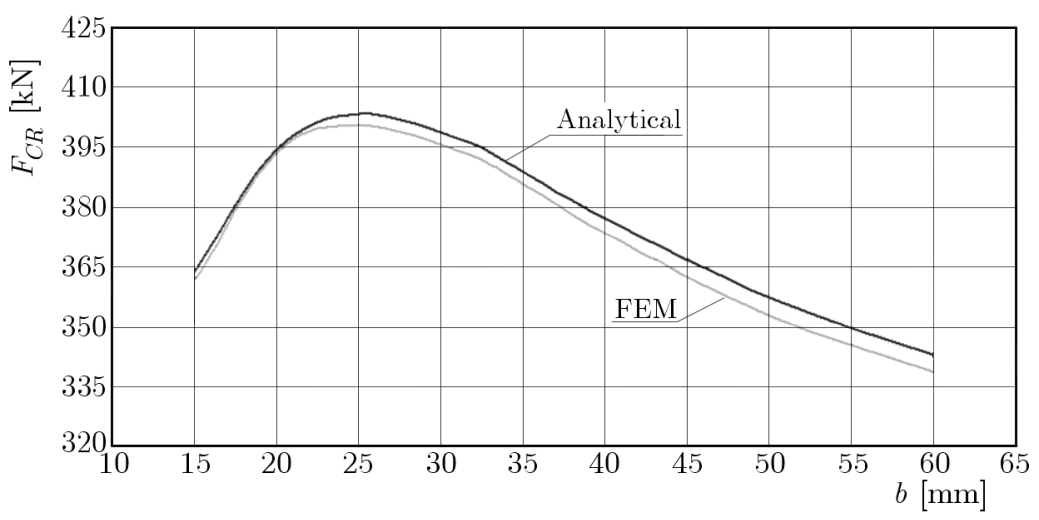

Fig. 14. Comparison of the critical values obtained from the analytical and numerical (FEM) methods for lateral buckling 


\section{Conclusions}

Theoretical studies of the buckling problem of the symmetrical triangular frame carried out on the basis of the analytical and numerical FEM models allow one to conclude that:

- flat buckling of the frame occurs when the width $b$ of the arm is small $(b \leqslant 18 \mathrm{~mm})$,

- lateral buckling of the frame occurs when the width $b$ of the arm is greater $(18 \mathrm{~mm} \leqslant b)$,

- maximum of the critical load exists for the width $b=25 \mathrm{~mm}$,

- the analytical model of the frame accurately describes the flat and lateral buckling phenomena. The analytical and FEM results comply with each other.

The presented study, considering the lateral buckling, has not been undertaken before.

\section{References}

1. Budiansky B., 1974, Theory of buckling and post-buckling behaviour of elastic structures, Advances in Applied Mechanics, 14, 1-65

2. BaŽAnt Z.P., Cedolin L., 1991, Stability of Structures. Elastic, Inelastic, Fracture, and Damage Theories, Oxford University Press, New York, Oxford

3. Chen W.F., Liu E.M., 1987, Structural Stability: Theory and Implementation, Elsevier, New York

4. Horne M.Z., Merchant W., 1965, The Stability of Frames, Pergamon Press, New York

5. Kaveh A., Salimbahrami B., 2007, Buckling load of symmetric plane frames using canonical forms, Computers and Structures, 85, 1420-1430

6. Magnucki K., Milecki S., 2012, Elastic stability of a brake triangle (in Polish), Modelowanie Inżynierskie, 44, 199-208

7. Simitses G.J., Hodges D.H., 2006, Fundamentals Structural Stability, Butterworth-Heinemann, an imprint of Elsevier

8. ŞAKAR G., Öztürk, SABuncu M., 2012, Dynamic stability of multi-span frames subjected to periodic loading, Journal of Constructional Steel Research, 70, 65-70

9. SobAś M., 2010, New generation of brake triangles for freight care (in Polish), Pojazdy Szynowe, 3, 21-30

10. Thompson J.M.T., Hunt G.W., 1973, A General Theory of Elastic Stability, John Wiley \& Sons, London, New York, Sydney, Toronto

11. Van Der Heijden A.M.A. (Editor), 2008, W.T. Koiter's Elastic Stability of Solids and Structures, Cambridge University Press, Cambridge, New York, Melbourne, Madrid, Cape Town, Singapore, São Paulo 